\title{
Impact of gallium concentration in the gas phase on composition of InGaAsN alloys grown by AP-MOVPE correlated with their structural and optical properties
}

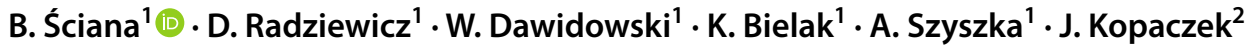

Received: 10 May 2019 / Accepted: 4 August 2019 / Published online: 10 August 2019

(c) The Author(s) 2019

\begin{abstract}
This work presents the epitaxial growth and material properties of InGaAsN epilayers obtained by atmospheric pressure metal organic vapour phase epitaxy. The main goal was to obtain InGaAsN quaternary alloys lattice-matched to GaAs in order to apply them as an intrinsic thick absorber in p-i-n solar cells. It allows improvement of their photovoltaic parameters (e.g. short circuit current, open circuit voltage) by reducing the density of misfit dislocations. To overcome the main difficulties connected with achieving InGaAsN composition with $I n / N$ ratio of $\sim 3$, which guarantees a lattice matching to GaAs, epitaxial processes were carried out with different concentration of gallium source in the gas phase. Diffraction curves, measured using HRXRD, indicated that the main aim of this work was achieved for the gas molar ratio $G a /(G a+I n)=0.935$. The optical quality and surface morphology of the investigated structures examined by PL, CER and AFM methods are also presented and discussed.
\end{abstract}

\section{Introduction}

The unique properties of dilute nitrides, such as GaAsN or InGaAsN with small concentrations of nitrogen, arise from a difference in size between $\mathrm{N}$ and As atoms, and a large electronegativity of $\mathrm{N}$ compared to $\mathrm{Ga}$, In and As. These semiconductors reveal a negative and immense bowing coefficient (up to $40 \mathrm{eV}$ ) dependent on composition [1], a large conduction band offset and an effective electron mass comparable to holes [2]. Dilute nitrides with a low band gap (about $1 \mathrm{eV}$ ) grown on GaAs or Ge substrates are beneficial for telecom emitters and efficient multijunction solar cells (MJSCs) with a very high photovoltaic conversion efficiency [3]. The epitaxial technology of dilute nitrides is highly complex, because even a small amount of nitrogen introduced into the GaAs or InGaAs crystalline structure strongly deteriorates their structural and optical

B. Ściana

beata.sciana@pwr.edu.pl

1 Faculty of Microsystem Electronics and Photonics, Wrocław University of Science and Technology, 11/17 Janiszewskiego St, 50-372 Wrocław, Poland

2 Faculty of Fundamental Problems of Technology, Wrocław University of Science and Technology, Wybrzeże Wyspiańskiego 27, 50-370 Wrocław, Poland properties. In addition, the nitrogen incorporation decreases minority carrier diffusion lengths, which degrades the performance of solar cells. Moreover, these layers are almost exclusively grown by the molecular beam epitaxy (MBE) $[4,5]$, or low pressure metal organic vapour phase epitaxy (LP-MOVPE) [6-9]. Therefore, the growth of dilute nitrides has been scarcely studied in an atmospheric pressure metal organic vapour phase epitaxy (AP-MOVPE) [10, 11], which is a challenge for growers, but allows the reduction of the production cost of MJSCs based on these metastable compounds. Our earlier experiments [12-15] indicated a high In/ $\mathrm{N}$ composition ratio $(10 \div 30)$ in InGaAsN alloys grown by AP-MOVPE. Consequently it was impossible to obtain thick $(\sim 1 \mu \mathrm{m})$ layers with good material quality. In this paper, we present the first results regarding optimization of gallium source concentration in the gas phase, in order to obtain InGaAsN epilayers lattice-matched to $\mathrm{GaAs}$ and $\mathrm{Ge}$ substrates for their application in $\mathrm{p}$-i-n solar cell constructions, with a thick absorption region. The influence of the $G a /(G a+I n)$ molar ratio in the gas phase on structural and optical properties of the investigated $\mathrm{InGaAsN} / \mathrm{GaAs}$ heterostructures is presented and discussed. 


\section{Experimental details}

The undoped InGaAsN/GaAs heterostructures were grown on Si-doped and semi-insulating (SI) GaAs ( $\left.\begin{array}{lll}1 & 0 & 0\end{array}\right)$ substrates with misorientation of $\pm 0.05 \div 0.08^{\circ}$ toward $\left[\begin{array}{lll}1 & 1 & 0\end{array}\right]$ in a horizontal AP-MOVPE AIX200 R\&D Aixtron reactor. Trimethylgallium (TMGa), trimethylindium (TMIn), tertiarybutylhydrazine (TBHy) and a 10\% mixture of arsine $\left(\mathrm{AsH}_{3}\right)$ in $\mathrm{H}_{2}$ were used as growth precursors, while a high purity $\mathrm{H}_{2}$ was used as a carrier gas. Test structures consisted of $400 \mathrm{~nm}$ thick GaAs buffer and of $\sim 100 \mathrm{~nm}$ thick InGaAsN epilayers. Constant process parameters during growth of InGaAsN were: the growth temperature $T_{g}=585{ }^{\circ} \mathrm{C}$ and the molar ratio of nitrogen source to the total group-V precursors in a gas phase $N /(N+A s)=0.730$. The GaAs buffer layer was deposited at the optimal growth conditions $\left(T_{g}=670{ }^{\circ} \mathrm{C}\right.$ and $V / I I I$ ratio of 68$)$. In order to achieve the required $I n / N$ composition ratio of $\sim 3$, the gas phase molar ratio of gallium to the total amount of group-III precursors $G a /(G a+I n)$ was only increased from 0.821 to 0.970 by changing the flow rate of TMGa $V_{T M G a}=13.8,29.5,43.2,59,78.6,98.3 \mu \mathrm{mol} / \mathrm{min}$ (samples NI129, NI135, NI136, NI130, NI131, NI132) at the constant flow rate of TMIn $V_{T M I n}=3 \mu \mathrm{mol} / \mathrm{min}$. We expected both a decrease of indium incorporation and an increase of the growth rate, which could result in increasing the nitrogen content in the growing InGaAsN alloys $[12,16]$.

The structural properties of the investigated InGaAsN/ GaAs heterostructures were measured by means of a high resolution X-ray diffraction (HRXRD) and an atomic force microscopy (AFM). HRXRD studies were performed using a PANalytical X'Pert Pro high-resolution X-ray diffractometer in the following configuration: $\mathrm{Cu} \mathrm{K}_{\alpha 1}$ $(\lambda \simeq 1.5406 \AA$ ) radiation, four-bounce Ge (4 0 0) hybrid monochromator and three-bounce Ge (2 20$)$ analyzer. The diffraction measurements of the $\omega / 2 \theta$ curves and reciprocal space maps (RSMs) were performed in the triple-axis geometry. The surface morphology of InGaAsN epilayers was observed in the air by means of the Bruker Multimode $\mathrm{V}$ atomic force microscope, using the tapping mode and a standard silicon probe (nominal tip radius $<10 \mathrm{~nm}$ ). The optical peculiarities were examined on the basis of photoluminescence (PL) and contactless electro-reflectance (CER) spectroscopies, both carried out at room temperature. Photoluminescence spectra were measured using a YAG laser operating at $532 \mathrm{~nm}$ with the optical power of $60 \mathrm{~mW}$. The contactless electro-reflectance spectroscopy was applied to determine the band gap energy of the investigated InGaAsN epilayers, to check if the desired value of approximately $1 \mathrm{eV}$ was achieved, as well as to support an analysis of the measured diffraction rocking curves. This method is a classical modulation spectroscopy, in which the electric field inside the sample is modulated by an external electric field, which leads to changes in optical reflectance (the sample is placed in a light transparent capacitor). The thickness and composition of all InGaAsN epilayers were determined from the simulations of the measured diffraction curves for the symmetrical ( 00 4) reflection, taking into account the bandgap value evaluated from the CER spectra. Detailed information about the algorithm used was previously published [17, 18].

\section{Results and discussion}

All obtained InGaAsN/GaAs heterostructures were investigated by means of HRXRD, AFM, PL and CER techniques, and the following sections contain the obtained results and discussion.

\subsection{Calibration curves}

The dependencies of the composition (In and $\mathrm{N}$ contents in a solid phase) and growth rate (GR) of InGaAsN epilayers on the $G a /(G a+I n)$ molar ratio determined on the basis of simulation of the measured diffraction curves are depicted in Fig. 1.

According to our expectations, the incorporation of indium drastically decreases, while nitrogen concentration increases with increasing gallium concentration in the gas phase. For the highest value of $G a /(G a+I n)=0.970$, indium content in InGaAsN alloy is negligible, while nitrogen content increases more than double. For the lower values of $G a /(G a+I n)=0.821,0.908,0.935$ (samples NI129, NI135, NI136), InGaAsN epilayers reveal a composition inhomogeneity [19-25] typical for dilute nitrides, with a linear increase of indium content toward the sample surface. In the case of the growth rate, the obtained dependence is more complex (Fig. 1b). Initially, the value of $G R$ decreases with increasing $\mathrm{Ga}$ concentration in the gas phase, while above the value of $G a /(G a+I n)=0.93 \div 0.94$ slightly increases. It shows that the epitaxial growth of InGaAsN alloys is mainly promoted by In incorporation (more probability of InGaAs crystallization than GaAsN), which confirms a very low $\mathrm{N}$ incorporation efficiency in GaAs under the applied growth conditions. The $G R$ and composition for all the investigated structures are summarized in Table 1. In the case of the samples NI129, NI135 and NI136, the values of $I n / N$ ratio were calculated for the highest values of In content located at the surface, which has the greatest effect on the stresses generated in the InGaAsN layer. 
(a)

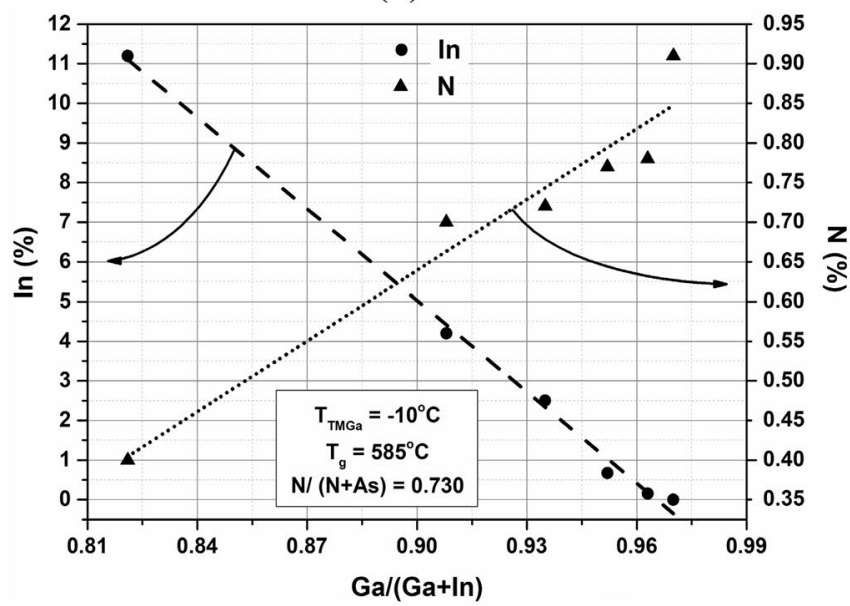

(b)

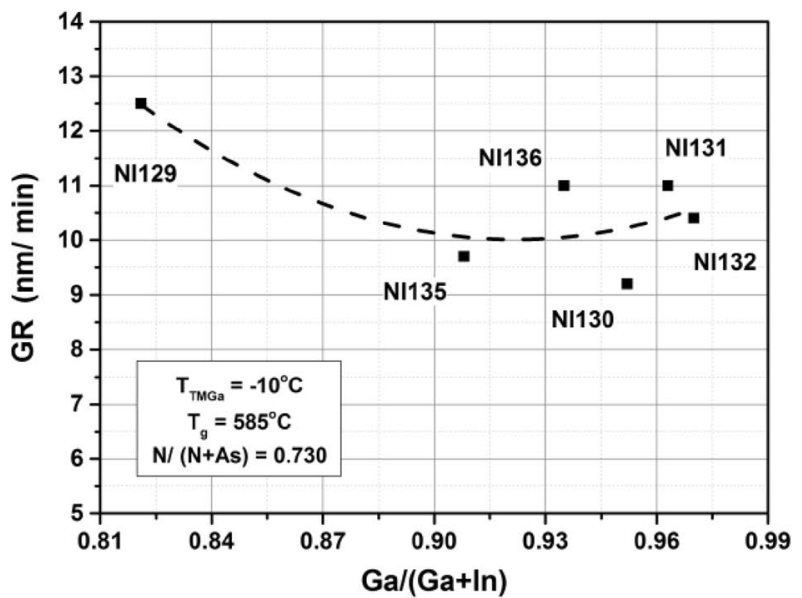

Fig. 1 The influence of the $G a /(G a+I n)$ molar ratio on composition (a) and growth rate (b) of investigated InGaAsN epilayers

Table 1 Growth rates and composition of investigated InGaAsN epilayers obtained for different $G a /(G a+I n)$ molar ratios

\begin{tabular}{|c|c|c|c|c|c|c|}
\hline $\begin{array}{l}\text { Technological/ } \\
\text { material param- } \\
\text { eter }\end{array}$ & Sample NI129 & Sample NI135 & Sample NI136 & Sample NI130 & Sample NI131 & Sample NI132 \\
\hline$G a /(G a+I n)$ & 0.821 & 0.908 & 0.935 & 0.952 & 0.963 & 0.970 \\
\hline$G R(\mathrm{~nm} / \mathrm{min})$ & 12.5 & 9.7 & 11 & 9.2 & 11 & 10.4 \\
\hline Composition & $\begin{array}{l}\mathrm{In}=7.5 \div 11.2 \% \\
\mathrm{~N}=0.4 \%\end{array}$ & $\begin{array}{l}\text { In }=0.3 \div 4.8 \% \\
N=0.7 \%\end{array}$ & $\begin{array}{l}\text { In }=0.5 \div 2.8 \% \\
\mathrm{~N}=0.7 \%\end{array}$ & $\begin{array}{l}\mathrm{In}=0.67 \% \\
\mathrm{~N}=0.77 \%\end{array}$ & $\begin{array}{l}\mathrm{In}=0.15 \% \\
\mathrm{~N}=0.78 \%\end{array}$ & $\begin{array}{l}\mathrm{In}=0 \% \\
\mathrm{~N}=0.91 \%\end{array}$ \\
\hline $\operatorname{In} / \mathrm{N}$ & 28 & 6.86 & 4 & 0.87 & 0.19 & 0 \\
\hline
\end{tabular}

Bold value indicates the parameters of InGaAsN layer the best lattice-matched to GaAs

(a)

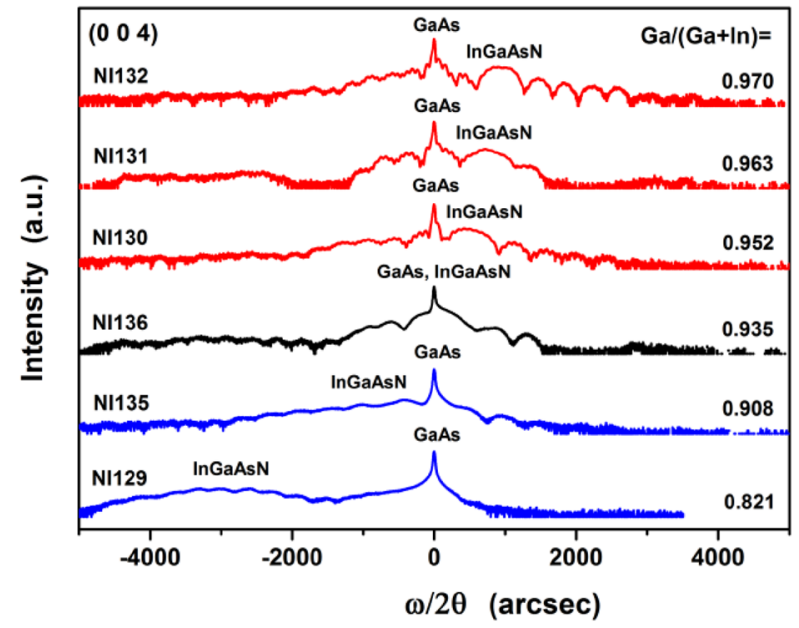

(b)

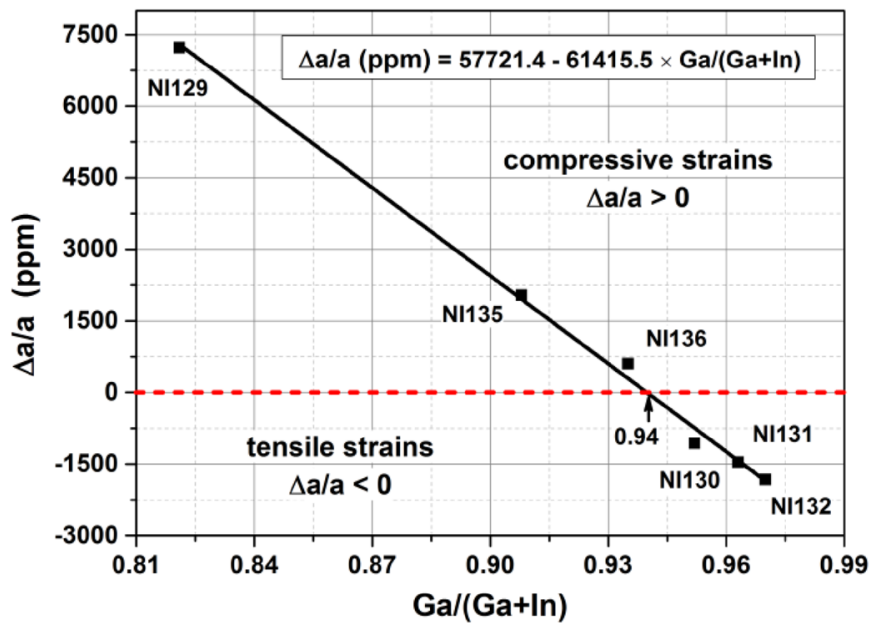

Fig. 2 The measured diffraction curves for $\left(\begin{array}{lll}0 & 0 & 4\end{array}\right)$ reflection of InGaAsN/GaAs heterostructures grown at different gas $G a /(G a+I n)$ molar ratios (a) and the dependence of the lattice mismatch of InGaAsN layers on the $G a /(G a+I n)$ molar ratio (b) 


\subsection{Structural properties}

The obtained diffraction curves of the samples grown at the gas $G a /(G a+I n)$ molar ratio changed from 0.821 to 0.970 are shown in Fig. 2a. For the lowest $G a /(G a+I n)$ values of 0.821 and 0.908 (samples NI129, NI135; blue curves), which favours higher In incorporation, the broad and blurred signal corresponding to the InGaAsN layer is placed to the left (for the smaller Bragg angles) of the main high intensity peak coming from the GaAs substrate. It indicates that the lattice parameter of these InGaAsN epilayers is greater than the GaAs which corresponds to $I n / N$ ratio higher than $3(\mathrm{In} / \mathrm{N}=3$ guarantees a lattice match to GaAs [3]). For $\mathrm{Ga} /$ $(G a+I n)>0.908$ (samples NI130-132 and NI136, red and black curves in Fig. 2a) the indium incorporation decreases, and the InGaAsN-related signal shifts to the bigger Bragg angles towards the GaAs substrate. For sample NI136, it is nearly overlapped with GaAs, while for $G a /(G a+I n)>0.935$ (samples NI130, NI131, NI132), it appears distinctly to the right of the GaAs peak. This means the last three mentioned samples have the lattice parameter smaller than the GaAs which corresponds to $I n / N$ ratio below 3 . The simulations of the measured rocking curves of the compressive strained InGaAsN alloys with the highest In content (samples NI 129, NI135, NI136) reveal a composition gradation $[20,24,25]$ typical for dilute nitrides. The indium content increases linearly toward the sample surface, while the $\mathrm{N}$ content remains constant (Table 1, subsection 3.1). This tendency is not observed for the tensile strained InGaAsN with In content below 1\% (samples NI130, NI131, NI132), which exhibit a homogeneous composition. Figure $2 \mathrm{~b}$ presents the dependence of the lattice mismatch $\Delta a / a$ (where $\Delta a$ is a difference between InGaAsN layer lattice parameter and GaAs substrate lattice parameter $a$ ), determined from the rocking curves for all investigated samples on the $G a /(G a+I n)$ molar ratio. The best lattice matching of InGaAsN to GaAs was obtained for the $G a /(G a+I n)$ molar ratio of 0.935 (sample NI136). This layer is compressively strained with the lattice mismatch $\Delta a / a=605.61 \mathrm{ppm}$. A linear approximation of the obtained results (the equation is shown in Fig. 2b) allowed estimation of the value of $G a /(G a+I n)=0.94$, which guarantees the lattice matching of InGaAsN to GaAs (the zero place of the determined linear function, $\Delta a=0$ ).

Figure 3 presents the reciprocal space maps around the asymmetrical reflection (2 24 ) of heterostructures containing InGaAsN with the highest In content (sample NI129), InGaAsN with the best lattice-matched to GaAs (sample NI136) and InGaAsN with the largest N content (sample NI132). They show that all investigated structures are fully strained. In the case of the most compressively strained InGaAsN layer (sample NI129, Fig. 3a), a diffused scattering along the horizontal axis representing the in-plane scattering vector component $Q_{x}$ is distinctly visible. It may be caused by the presence of structural defects [26] or morphological peculiarities. This scattering was described by full width at half maximum ( $F W H M)$ of main peak, determined from (lll $\left.0 \begin{array}{ll}0 & 4\end{array}\right)$ symmetrical space maps reflection (not included here). For sample NI129, the highest value of 12 arc sec was obtained, while only 7 arc sec revealed the samples NI136 and NI132. It confirms the results coming from AFM measurements, which showed the worst surface roughness of sample NI129, which is probably connected with 2-D and/ or 3-D island growth modes.

\subsection{AFM analysis}

The main aim of the AFM measurements was to evaluate the surface roughness of the investigated structures depending (a)

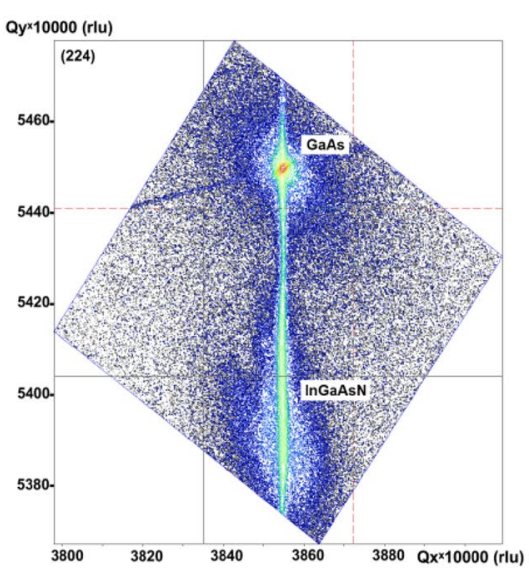

(b)

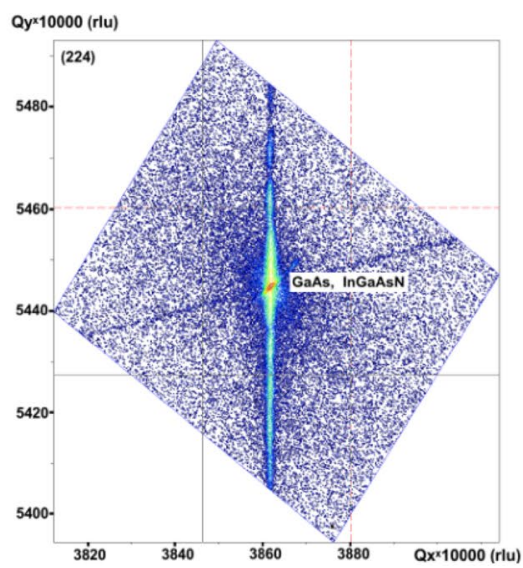

(c)

NI132

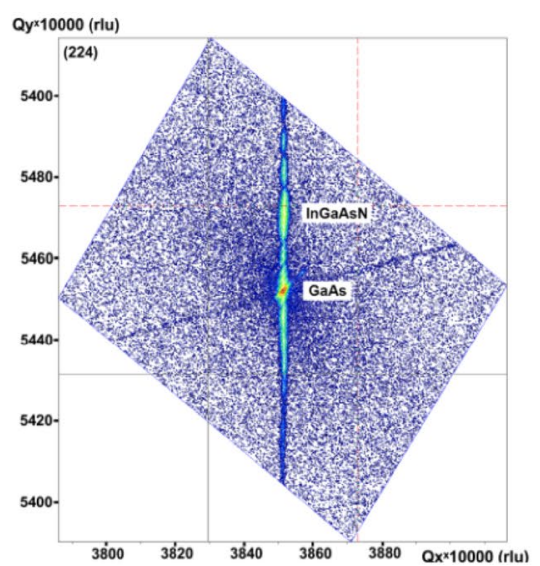

Fig. 3 Reciprocal space maps around the asymmetrical reflection (2 24 ) of InGaAsN/GaAs heterostructures grown at different $G a /(G a+I n)$ ratios: a 0.821 (sample NI129); b 0.935 (sample NI136) and c 0.970 (sample NI132) 
on the gas $G a /(G a+I n)$ molar ratio, which directly corresponds to In and $\mathrm{N}$ contents in the InGaAsN solid phase, the composition inhomogeneity and the induced local strains. Typical for dilute nitrides growth conditions: low growth temperatures, a small amount of $\mathrm{AsH}_{3}$ together (in our case) with the use of the atmospheric pressure process, preventing the step-flow mode growth and favouring quasitwo-dimensional (2-D) or three-dimensional (3-D) growth modes [27-30].

Figure 4 shows the AFM images $(10 \times 10,5 \times 5$ and $2 \times 2 \mu \mathrm{m}^{2}$ scans) and the evaluated root mean square roughness $\left(R_{R M S}\right)$ for: the reference GaAs layer (Fig. 4a) grown under the same conditions as GaAs buffer in the test structures, InGaAsN with the highest In content (sample NI129, the greatest compressive strains-Fig. 4b), InGaAsN lattice-matched to GaAs (sample NI136, the smallest value of the introduced stress-Fig. 4c) and InGaAsN with the largest $\mathrm{N}$ content and negligible In content (sample NI132, the greatest tensile strains-Fig. 4d). The surface morphology of the investigated InGaAsN epilayers distinctly differs in comparison to the reference GaAs layer with a very smooth $\left(R_{R M S}=1.69 \div 2.04 \AA\right)$ step/terrace structure, characteristic of the step-flow mode growth. In the case of InGaAsN, the visible surface features indicate 2-D/3-D island formation on terraces and a different way of their subsequent coalescence. As previously mentioned, this island structure is the most likely for our InGaAsN growth parameters as: a low growth temperature $\left(T_{g}=585{ }^{\circ} \mathrm{C}\right)$ resulting in a small surface adatom diffusion length, a low $\mathrm{AsH}_{3}$ concentration in the gas phase $(A s /(A s+N)$ molar ratio of (a)

GaAs

$10 \times 10 \mu \mathrm{m}^{2}, \quad R_{R M S}=1.69 \AA$

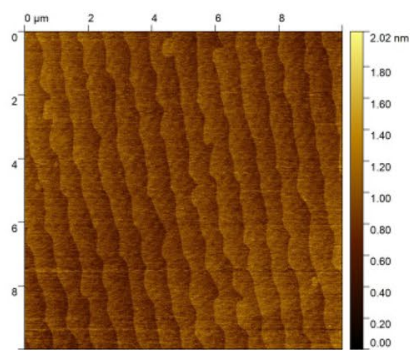

$5 \times 5 \mu \mathrm{m}^{2}, R_{R M S}=1.80 \AA$

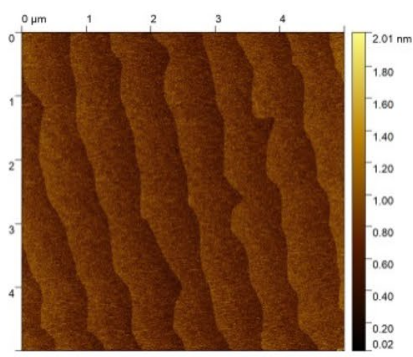

$2 \times 2 \mu \mathrm{m}^{2}, R_{R M S}=2.04 \AA$

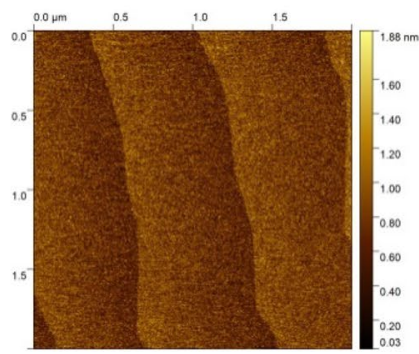

(b) InGaAsN - NI129

$10 \times 10 \mu \mathrm{m}^{2}, R_{R M S}=17.50 \AA$

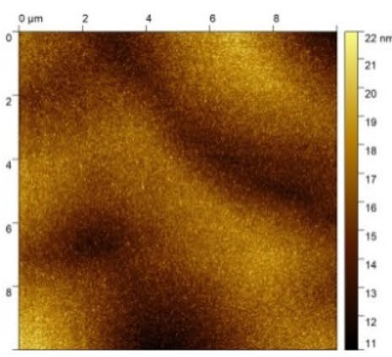

$5 \times 5 \mu \mathrm{m}^{2}, R_{R M S}=15.46 \AA$

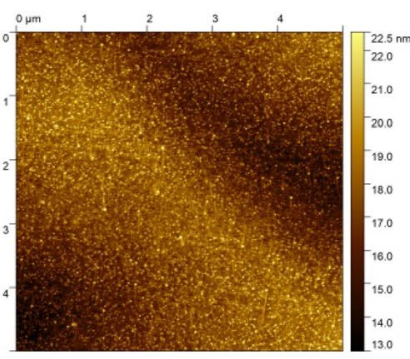

$2 \times 2 \mu \mathrm{m}^{2}, R_{R M S}=11.70 \AA$

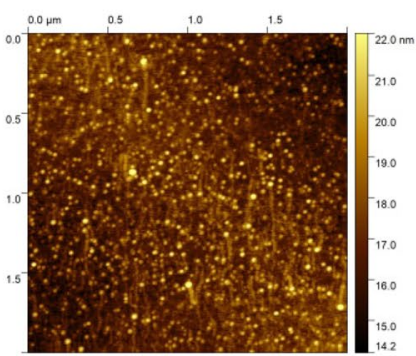

(c) InGaAsN - NI136

$10 \times 10 \mu \mathrm{m}^{2}, R_{R M S}=11.83 \AA$

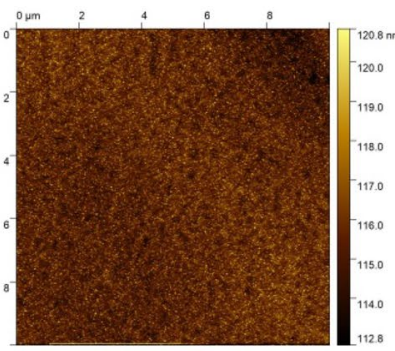

$5 \times 5 \mu \mathrm{m}^{2}, R_{R M S}=10.26 \AA$

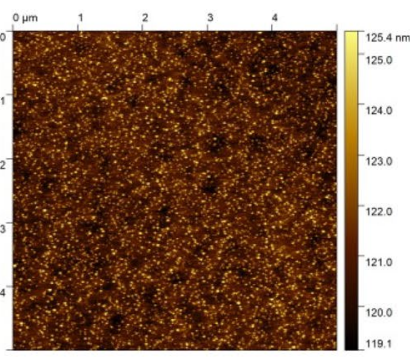

$2 \times 2 \mu \mathrm{m}^{2}, R_{R M S}=10.05 \AA$

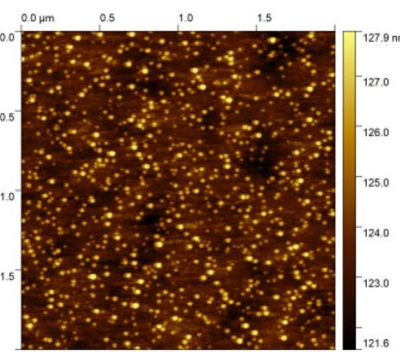

(d) InGaAsN - NI132

$$
10 \times 10 \mu \mathrm{m}^{2}, R_{R M S}=11.07 \AA
$$

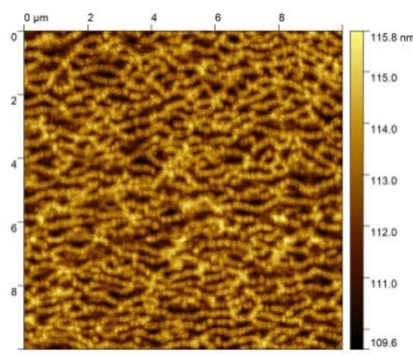

$5 \times 5 \mu \mathrm{m}^{2}, R_{R M S}=10.95 \AA$

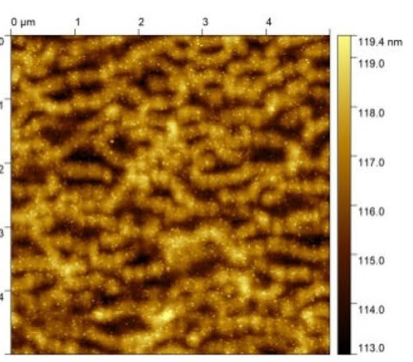

$2 \times 2 \mu \mathrm{m}^{2}, R_{R M S}=10.43 \AA$

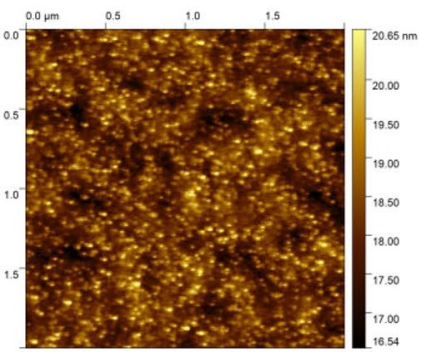

Fig. 4 AFM images and evaluated the $R_{R M S}$ roughness for: a GaAs reference sample; b-d InGaAsN epilayers grown at the gas $G a /(G a+I n)$ molar ratios of $0.821,0.935$ and 0.970 , respectively 
0.27 ) and the use of the atmospheric pressure MOVPE process, causing a higher amount of incorporated impurities during InGaAsN growth (mainly organic precursor debris). Additionally, a small misorientation of GaAs substrates $\left( \pm 0.05 \div 0.08^{\circ}\right)$ causes a large step separation, which also favours the 2-D nucleation on the middle area of the terraces [27]. All InGaAsN surfaces show a high density of nanometer-size islands initiating the nucleation process. Their height $(4.0 \pm 0.5 \mathrm{~nm})$ and diameter $(35 \pm 2 \mathrm{~nm})$ were determined based on detailed AFM imaging performed with a supersharp AFM tip (radius $<2 \mathrm{~nm}$ ) in order to minimize the effect of a tip shape on the measured surface features. The exemplary $150 \times 150 \mathrm{~nm}^{2}$ surface image of sample NI136 and the measured profiles used for calculating the islands' dimension are presented in Fig. 5a and b, respectively.

The formation of these islands can be connected with the precursor debris [12] and/or the disrupted step edges [27, 28]. In the case of the compressively strained InGaAsN layer (sample NI129, Fig. 4b), elongated bulges (about $0.5 \mu \mathrm{m}$ in length) beside these islands are also visible. The surface roughness $R_{R M S}$ changes from $11.7 \AA\left(2 \times 2 \mu \mathrm{m}^{2}\right.$ scan $)$ to $17.5 \AA\left(10 \times 10 \mu \mathrm{m}^{2} \mathrm{scan}\right)$, which indicates the 2-D and/or 3 -D island growth mode. The elongated shape of the formed islands can be connected with a higher mobility and a higher surface-diffusion length of the $\mathrm{Ga}$ and In adatoms (the lowest $G a /(G a+I n)$ ratio of 0.821) [27, 29]. The surface morphology of lattice-matched InGaAsN (sample NI136, Fig. 4c), grown at a higher $G a /(G a+I n)$ molar ratio of 0.935 , shows a mesh-like structure described in [30]. Firstly, they are locally formed and randomly distributed islands, which in the second stage join together to create the observed mesh morphology. The $R_{R M S}$ roughness is varied from $10.05 \AA$ $\left(2 \times 2 \mu \mathrm{m}^{2}\right.$ scan $)$ to $11.83 \AA\left(10 \times 10 \mu \mathrm{m}^{2}\right.$ scan $)$, which is smaller in comparison to the most compressively strained
NI129 sample. The observable effect of increasing the root mean square roughness parameter with enlarging the AFM image size (which is most pronounced for NI129 sample) is connected with the roughness area dependence mechanism $[31,32]$. It shows the scaling behaviour of surface topography, when, by extending the sampling area, the larger-scale surface topography components are included in the $R_{R M S}$ parameter calculation, resulting in its increase. In the case of the highest $G a /(G a+I n)$ molar ratio of 0.970 , when the most tensile-strained InGaAsN was obtained with a negligible In content (probably the GaAsN epilayer with $\mathrm{N}$ content of $0.91 \%$ was grown based on HRXRD results), the surface morphology shows a slightly elongated network structure similar to tangled "spaghetti" (sample NI132, Fig. 4d). The evaluated $R_{R M S}$ roughness is comparable to the latticematched InGaAsN (samples NI136). The presented AFM studies suggest that the network morphology of InGaAsN epilayers created for the highest values of $G a /(G a+I n)$ is not directly connected with introduced strains, but is probably related to the decreased group III adatom surface mobility (diffusion-length) resulting from an increase of Ga concentration in the gas phase. It is also confirmed by the changes of the $R_{R M S}$ parameter for the samples NI129, NI136 and NI132 $\left(2 \times 2 \mu \mathrm{m}^{2}\right.$ and $5 \times 5 \mu \mathrm{m}^{2}$ scans $)$. With the increasing $G a /(G a+I n)$ molar ratio, the $R_{R M S}$ parameter firstly decreases, and then increases again. It can be explained by the competitive influence of the strains and group III adatoms surface mobility on InGaAsN layers roughness. The smaller roughness of sample NI136 in comparison to sample NI129 means that a distinct reduction of compressive strains for $G a /(G a+I n)=0.935$ has a greater impact on improvement of InGaAsN layer flatness than the lower adatoms mobility, in this case, worsens its surface quality. For sample NI132, grown at highest value of $G a /(G a+I n)=0.970$, the (a)

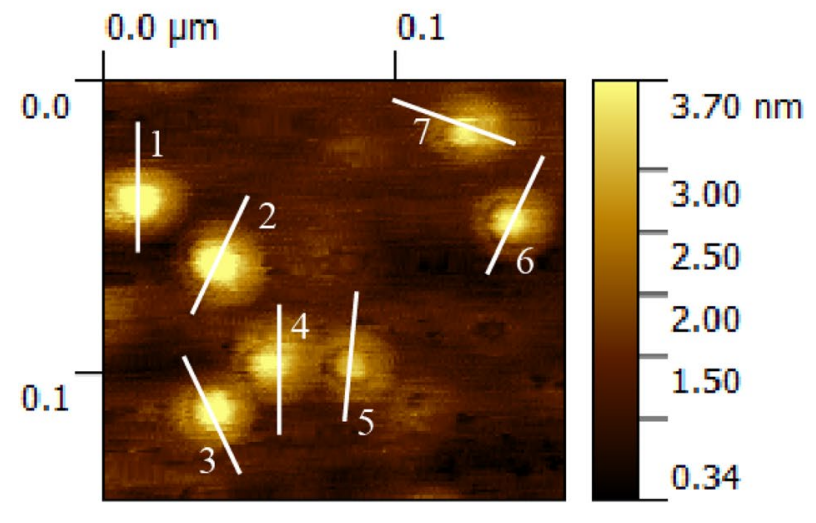

(b)

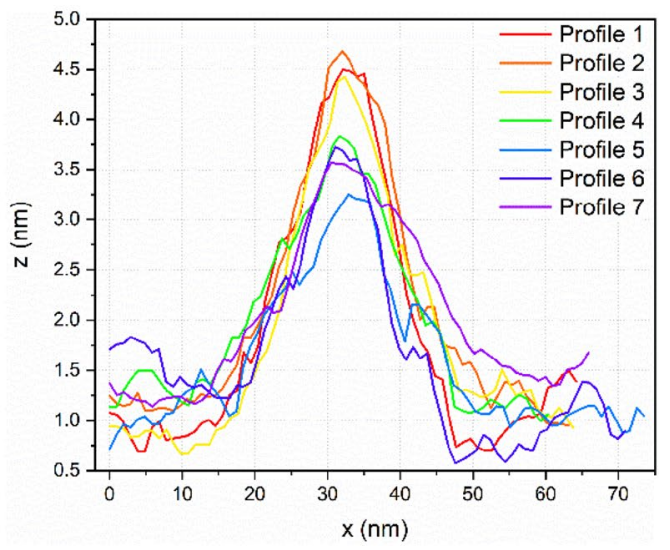

Fig. 5 The exemplary high resolution AFM surface image of the sample NI136 (a) and the measured profiles used for calculating of the islands dimension (b) 
$R_{R M S}$ parameter slightly increases in comparison to sample NI136, but remains smaller than for sample NI129. It means the tensile strains introduced into this layer (much lower than for sample NI129) have a negligible influence on the InGaAsN surface quality, and a formation of the network morphology with a higher roughness is related to exceedingly low group III adatoms mobility.

\subsection{Photoluminescence spectroscopy}

The obtained PL spectra reveal composition inhomogeneity (except of the samples NI135 and NI136, best latticematched to GaAs), visible as broad or splitting spectra shown in Fig. 6a. This inhomogeneity is typical for metastable alloys, such as dilute nitrides, and is spontaneously created during their growth, especially at higher process temperatures that enhance phase segregation [33]. This fact can be explained by a dominance of surface kinetics, the nonlinear dependence of $\mathrm{N}$ incorporation on In content [34], and of the strain gradient [25]. Both alloy content fluctuations and an inhomogeneous distribution of the N-related defects result in a band gap variation [35]. It is distinctly visible in the PL spectra recorded for InGaAsN with the highest In (sample NI129) and N (sample NI132) contents-Fig. 6b. They exhibit overlapping emissions: a main PL peak at about $1.21 \div 1.22 \mathrm{eV}$ (band gap-related emission), and some response at about $1.16 \div 1.17 \mathrm{eV}$ (labeled as $E_{2}$ in Fig. 6b). The broadening of PL spectra $F W H M_{P L}$ is the largest $(75 \div 79 \mathrm{meV})$ for the structures with the highest $\mathrm{N}$ content (samples NI130 $\div$ NI132), which results from a (a)

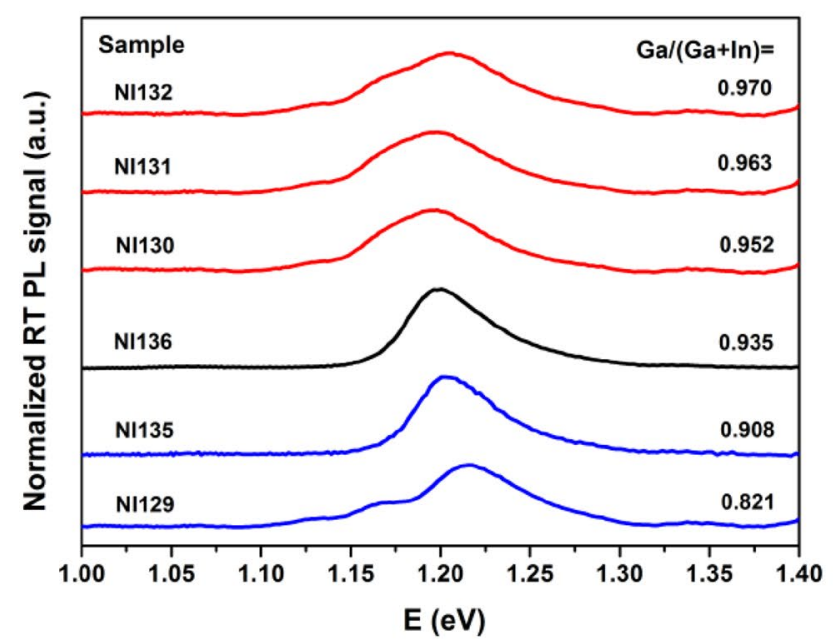

(b)

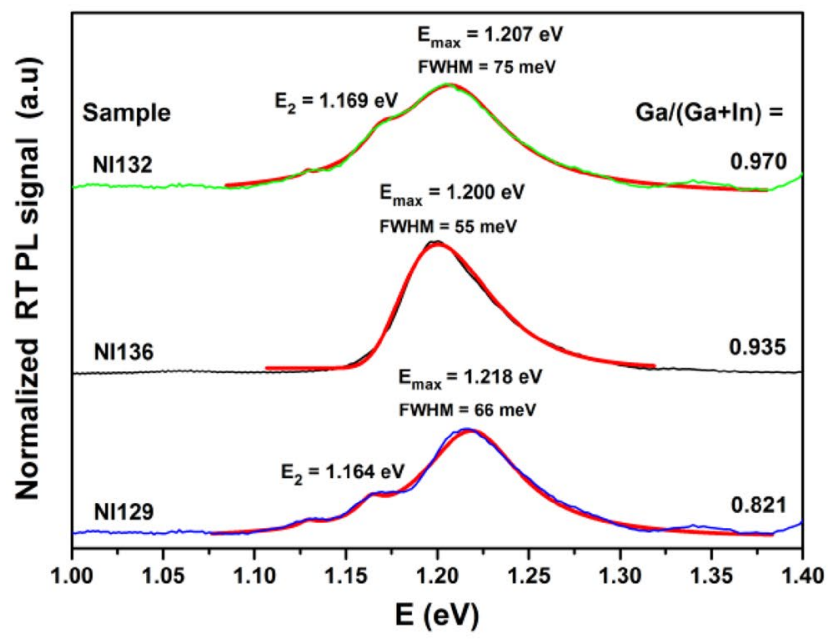

(c)

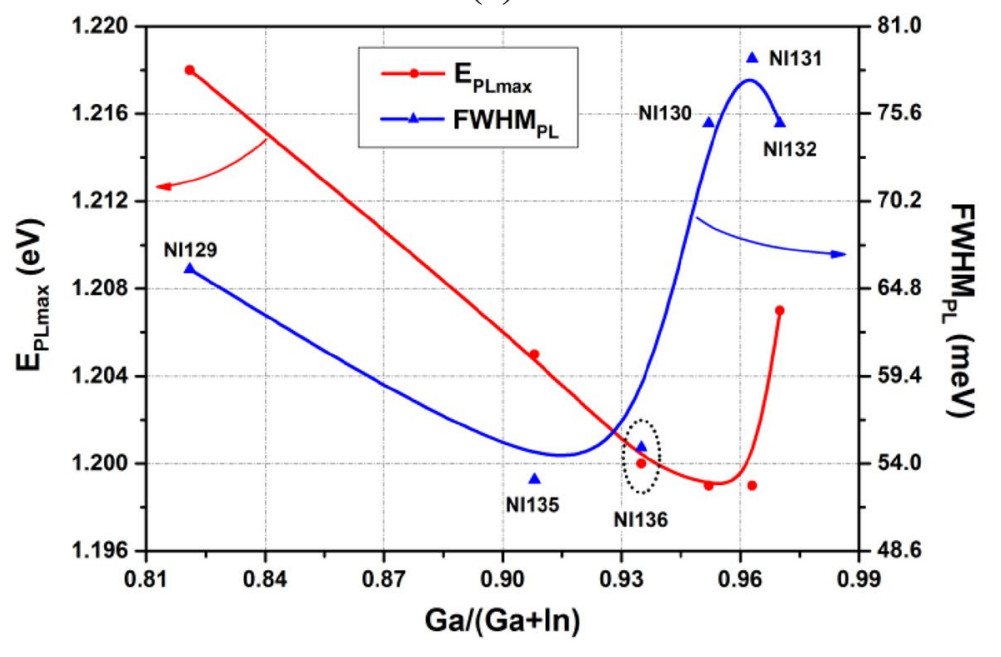

Fig. 6 RT PL spectra recorded for all investigated InGaAsN/GaAs heterostructures (a); RT PL spectra of InGaAsN grown at the $\mathrm{Ga}$ / $(G a+I n)$ molar ratios of $0.821,0.935,0.970$ (b) and a relation between the parameters evaluated from RT PL spectra- $E_{P L m a x}$ and $F W H M_{P L}$ and the applied $G a /(G a+I n)$ molar ratios $(\mathbf{c})$ 
broad emission $\left(E_{2}\right)$, probably connected with a high density of N-related states localized close to the conduction band minimum [35]. In the case of sample NI129 with the highest In content, the value of $F W H M_{P L}$ is smaller $(66 \mathrm{meV})$, and a distinct split between the main emission signals is clearly visible, which suggests an influence of a phase separation effect.

The PL spectrum of the InGaAsN layer, the best latticematched to GaAs presented in Fig. 6b (sample NI136), shows a well-resolved band gap-related emission at $1.2 \mathrm{eV}$, with a small broadening $\left(F W H M_{P L}=55 \mathrm{meV}\right)$. The dependence of the parameters evaluated from the PL spectra $\left(E_{P L m a x}\right.$ - the energy of PL signal maximum and $F W H M_{P L}-$ the PL signal broadening) on the applied $G a /(G a+I n)$ molar ratio is presented in Fig. 6c. All determined parameters are listed in Table 2.

\subsection{Contactless electro-reflectance spectroscopy}

The measured CER spectra of the test InGaAsN/GaAs heterostructures recorded at room temperature (RT) are presented in Fig. 6a. They exhibit, besides distinct optical transitions related to GaAs at energy of $1.43 \mathrm{eV}$, the weak resonances detected at about $1.2 \mathrm{eV}$ associated with the InGaAsN layer. Additionally, CER spectra related to InGaAsN with three specific composition ratios: $I n / N=28$ (the highest In content, sample NI129), $I n / N$ ratio $=4$ (the best lattice-matched to GaAs, sample NI136) and $I n / N=0$ (indium absence, sample NI132), are shown in Fig. 7b.

The band gap energy $\left(E_{g}\right)$ of the InGaAsN epilayers and CER transition broadening ( $\Gamma$ parameter) were estimated by fitting the corresponding resonances using a low-field electro-modulation Lorentzian line shape functional form, described in $[36,37]$. The values of the obtained parameters, as well as the calculated ratio of InGaAsN to GaAs CER signal amplitudes $A_{\text {InGaAsN }} / A_{\text {GaAs }}$, are listed in Table 3.

The InGaAsN epilayers with the highest and the lowest $I n / N$ composition ratios (the most lattice-mismatched to GaAs) have a very week CER signal, whereas the narrowest resonance reveals sample NI129 with the highest In content. A similar relationship was observed for the InGaAs structures with decreasing In content of 12.8, 8.9 and 3.4\%

Table 2 The parameters $\left(E_{P L m a x}, E_{2}\right.$ and $\left.F W H M_{P L}\right)$ determined from fitting RT PL spectra of InGaAsN/GaAs heterostructures grown at different molar ratios $G a /(G a+I n)$

\begin{tabular}{|c|c|c|c|c|c|c|}
\hline $\begin{array}{l}\text { Estimated parameters/composi- } \\
\text { tion ratio (gas phase molar ratio) }\end{array}$ & Sample NI129 & Sample NI135 & Sample NI136 & Sample NI130 & Sample NI131 & Sample NI132 \\
\hline$E_{P \max }(\mathrm{eV})$ & 1.218 & 1.205 & 1.200 & 1.199 & 1.199 & 1.207 \\
\hline$E_{2}(\mathrm{eV})$ & 1.164 & - & - & 1.173 & 1.174 & 1.169 \\
\hline$F W H M_{P L}(\mathrm{meV})$ & 66 & 53 & 55 & 75 & 79 & 75 \\
\hline $\operatorname{In} / N$ & 28 & 6.86 & 4 & 0.87 & 0.19 & 0 \\
\hline$(G a /(G a+I n))$ & $(0.821)$ & $(0.908)$ & $(0.935)$ & $(0.952)$ & $(0.963)$ & $(0.970)$ \\
\hline
\end{tabular}

Bold values indicate the parameters of $\mathrm{InGaAsN}$ layer the best lattice-matched to $\mathrm{GaAs}$

(a)

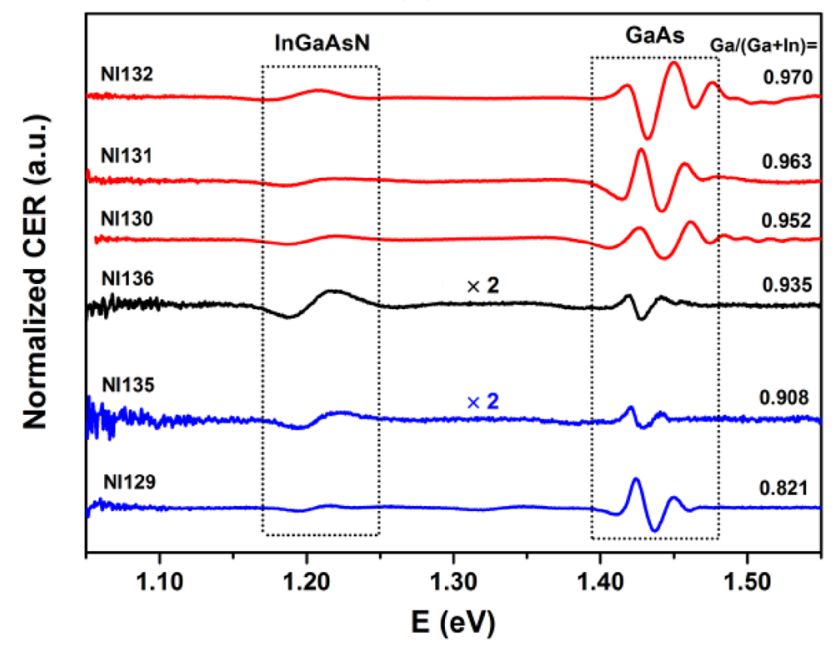

(b)

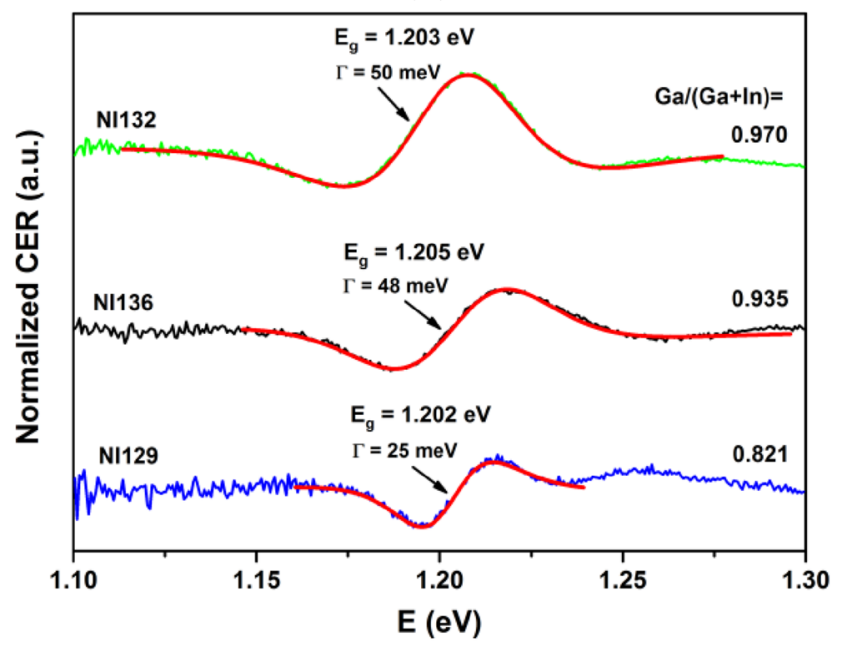

Fig. 7 The RT CER spectra of InGaAsN/GaAs haterostructures grown at: a all applied $G a /(G a+I n)$ molar ratios; $\mathbf{b}$ three chosen values of $G a /$ $(G a+I n)=0.821,0.935$, and 0.970 related to $I n / N$ composition ratio 28,4 , and 0 , respectively 
Table 3 Band gap energy $E_{g}, \Gamma$ parameter and ratio of CER signal amplitudes $A_{I n G a A s N} / A_{G a A s}$ determined from fitting RT CER spectra of investigated InGaAsN/GaAs heterostructures obtained for different $G a /(G a+I n)$ molar ratios

\begin{tabular}{lllllll}
\hline $\begin{array}{l}\text { Estimated parameters/composi- } \\
\text { tion ratio (gas phase molar ratio) }\end{array}$ & Sample NI129 & Sample NI135 & Sample NI136 & Sample NI130 & Sample NI131 & Sample NI132 \\
\hline$E_{g C E R}(\mathrm{eV})$ & 1.202 & 1.206 & $\mathbf{1 . 2 0 5}$ & 1.201 & 1.198 & 1.203 \\
$\Gamma(\mathrm{meV})$ & 25 & 39 & $\mathbf{4 8}$ & 52 & 53 & 50 \\
$A_{I n G a A s N} / A_{G a A s}$ & 0.175 & 0.766 & $\mathbf{1 . 1 5 4}$ & 0.232 & 0.145 & 0.121 \\
$I n / N$ & 28 & 6.86 & $\mathbf{4}$ & 0.87 & 0.19 & $(0.963)$ \\
$(G a /(G a+I n))$ & $(0.821)$ & $(0.908)$ & $\mathbf{( 0 . 9 3 5 )}$ & $(0.952)$ & $(0.970)$ \\
\hline
\end{tabular}

Bold values indicate the parameters of InGaAsN layer the best lattice-matched to GaAs

for which the values of $\Gamma$ parameter were 12.6, 13.9 and $17.2 \mathrm{meV}$, respectively [38]. Samples NI135 and NI136 (the best lattice-matched to GaAs) have the best optical properties, which exhibit the strongest InGaAsN-related transition with its broadening ( $\Gamma$ parameter) below $50 \mathrm{meV}$. The determined value of the InGaAsN band gap is about $1.2 \mathrm{eV}$ for all investigated heterostructures. Therefore, an increase of the gas phase molar ratio $G a /(G a+I n)$ allows to increase the $\mathrm{N}$ content and a growth of InGaAsN alloys lattice-matched to $\mathrm{GaAs}$, but further investigations are needed for lowering their band gap to the applications value of $1.0 \mathrm{eV}$, required for SCs.

\section{Conclusions}

The work presents the impact of the $G a /(G a+I n)$ molar ratio changed by gallium source concentration in the gas phase on the composition, growth rate and properties of InGaAsN alloys grown by atmospheric pressure metal organic vapour phase epitaxy. The main investigative task was to obtain InGaAsN epilayers lattice-matched to GaAs suitable for application in p-i-n solar cells with a thick absorption region. The analysis of the measured diffraction rocking curves confirmed our expectations that gallium concentration in the gas phase efficiently controls InGaAsN composition, resulting in In- or N-rich alloys. Based on the determined linear dependence of the lattice mismatch of InGaAsN on the $\mathrm{Ga}$ / $(G a+I n)$ ratio, the value of 0.94 was estimated for achieving a lattice matching to GaAs. AFM images showed a network morphology of InGaAsN grown at the highest values of $G a /(G a+I n)$ which is related to the decrease of group-III adatoms surface mobility. However, the worst surface roughness was obtained for InGaAsN with the highest In content grown at the lowest $G a /(G a+I n)$. PL and CER measurements revealed a good optical quality, and the lowest band gap of $1.2 \mathrm{eV}$ for InGaAsN layer the best lattice-matched to GaAs (sample NI136, $\Delta a / a=605.61 \mathrm{ppm}$ ). The obtained results coming from structural and optical studies suggest that a simultaneous increase in both the gallium and indium concentrations in the gas phase at the constant estimated value of $G a /(G a+I n)=0.94$ could result in the lowering of the InGaAsN band gap to $1 \mathrm{eV}$ required for photovoltaic applications. Thus, our future research will be focused on verifying this assumption.

Acknowledgements This work was co-financed by Wrocław University of Science and Technology statutory grants. The authors would like to thank I. Sankowska Ph.D., from the Łukasiewicz Research Network, Institute of Electron Technology (Warsaw) for the HRXRD measurements.

Open Access This article is distributed under the terms of the Creative Commons Attribution 4.0 International License (http://creativeco mmons.org/licenses/by/4.0/), which permits unrestricted use, distribution, and reproduction in any medium, provided you give appropriate credit to the original author(s) and the source, provide a link to the Creative Commons license, and indicate if changes were made.

\section{References}

1. U. Tisch, E. Finkman, J. Salzman, The anomalous bandgap bowing in GaAsN. Appl. Phys. Lett. 81, 463-465 (2002)

2. W.W. Chow, E.D. Jones, N.A. Modine, A.A. Allerman, S.R. Kurtz, Laser gain and threshold properties in compressive-strained and lattice-matched GaInNAs/GaAs quantum wells. Appl. Phys. Lett. 75, 2891-2893 (1999)

3. J.F. Geisz, D.J. Friedman, III-N-V semiconductors for solar photovoltaic applications. Semicond. Sci. Technol. 17, 769-777 (2002)

4. Y. Kamikawa-Shimizu, S. Niki, Y. Okada, Fabrication of homojunction GaInNAs solar cells by atomic hydrogen-assisted molecular beam epitaxy. Sol. Energy Mater. Sol. Cells 93(6-7), 1120-1123 (2009)

5. F. Langer, S. Perl, S. Höfling, M. Kamp, Graded band gap GaInNAs solar cells. Appl. Phys. Lett. 106, 233902 (2015)

6. K.S. Kim, S.J. Lim, K.H. Kim, J.R. Yoo, T. Kim, Y.J. Park, MOVPE growth optimization for optically efficient GaInNAs quantum well structure. J. Cryst. Growth 273(3-4), 368-374 (2005)

7. T.H. Wu, Y.K. Su, R.W. Chuang, C.Y. Huang, H.J. Wu, Y.C. Lin, 1-eV InGaAsN/GaAs quantum well structure for high efficiency solar application grown by MOVPE. J. Cryst. Growth 370, 236239 (2013)

8. T. Kim, A. Wood, H. Kim, Y. Kim, J. Lee, M. Peterson, Y. Sin, S. Moss, T.F. Kuech, S. Babcock, L.J. Mawst, Impact of Sb 
incorporation on MOVPE-grown "bulk" InGaAs(Sb)N films for solar cell application. IEEE J. Photovolt. 6(6), 1673-1677 (2016)

9. J.-N. Beaudry, R.A. Masut, P. Desjardins, GaAs1-xNx on GaAs(0 0 1): nitrogen incorporation kinetics from trimethylgallium, tertiarybutylarsine, and 1,1-dimethylhydrazine organometallic vaporphase epitaxy. J. Cryst. Growth 310, 1040-1048 (2008)

10. A. Ougazzaden, Y. Le Bellego, E.V.K. Rao, M. Juhel, L. Leprince, G. Patriarche, Metal organic vapor phase epitaxy growth of $\mathrm{GaAsN}$ on GaAs using dimethylhydrazine and tertiarybutylarsine. Appl. Phys. Lett. 70, 2861 (1997)

11. T. Hakkarainen, J. Toivonen, M. Sopanen, H. Lipsanen, GaInNAs quantum well structures for 1.55 um emission on GaAs by atmospheric pressure metalorganic vapor phase epitaxy. J. Cryst. Growth 234(4), 631-636 (2002)

12. M.C. López-Escalante, B. Ściana, W. Dawidowski, K. Bielak, M. Gabás, Atomic configurations in AP-MOVPE grown latticemismatched InGaAsN films unravelled by X-ray photoelectron spectroscopy combined with bulk and surface characterization techniques. Appl. Surf. Sci. 433, 1-9 (2018)

13. A. Kosa, L. Stuchlikova, L. Harmatha, M. Mikolasek, J. Kovac, B. Sciana, W. Dawidowski, D. Radziewicz, M. Tlaczala, Defect distribution in InGaAsN/GaAs multilayer solar cells. Sol. Energy 132, 587-590 (2016)

14. W. Dawidowski, B. Ściana, I. Zborowska-Lindert, M. Mikolášek, K. Bielak, M. Badura, D. Pucicki, D. Radziewicz, J. Kováč, M. Tłaczała, The influence of top electrode of InGaAsN/GaAs solar cell on their electrical parameters extracted from illuminated I-V characteristics. Solid-State Electron. 120, 13-18 (2016)

15. A. Kósa, L. Stuchlíková, W. Dawidowski, J. Jakuš, B. Ściana, D. Radziewicz, D. Pucicki, L. Harmatha, J. Kováč, M. Tłaczała, DLTFS investigation of InGaAsN/GaAs tandem solar cell. J. Elect. Eng. 65(5), 271-276 (2014)

16. Toivonen J (2003) Growth and properties of GaAsN structures, doctor's thesis, Helsinki University of Technology

17. D. Pucicki, K. Bielak, B. Ściana, W. Dawidowski, K. Żelazna, J. Serafińczuk, J. Kováč, A. Vincze, Ł. Gelczuk, P. Dłużewski, Structural characterization of doped thick GaInNAs layers-ambiguities and challenges. J. Elect. Eng. 65, 299-303 (2014)

18. D. Pucicki, K. Bielak, B. Ściana, D. Radziewicz, M. LatkowskaBaranowska, J. Kováč, A. Vincze, M. Tłaczała, Determination of composition of non-homogeneous GaInNAs layers. J. Cryst. Growth 433, 105-113 (2016)

19. H. Saito, T. Makimoto, N. Kobayashi, MOVPE growth of strained InGaAsN/GaAs quantum wells. J. Cryst. Growth 195(1-4), 416420 (1998)

20. M.C. Chan, C. Surya, P.K.A. Wai, The effects of interdiffusion on the subbands in GaxIn1 - xN0.04As0.96/GaAs quantum well for 1.3 and $1.55 \mu \mathrm{m}$ operation wavelengths. J. Appl. Phys. 90(1), 197-201 (2001)

21. Miyashita N, Ahsan N, Okada Y (2010) Antimony enhanced homogeneous nitrogen incorporation into GaInNAs films grown by atomic hydrogen-assisted molecular beam epitaxy. 2010 35th IEEE Photovoltaic Specialists Conference, IEEE; 2010 Jun; Available from: http://dx.doi.org/10.1109/pvsc.2010.5616583

22. T. Mori, T. Hanada, T. Morimura, G. Kobayashi, T. Yao, T. Miyajima, T. Uruga, Study of local segregation in GaInNAs using EXAFS measurements. J. Phys. Chem. Solids 69(2-3), 298-301 (2008)

23. A. Hashimoto, T. Yamaguchi, T. Suzuki, A. Yamamoto, Hall electron mobility versus N spatial distribution in III-V-N systems. J. Cryst. Growth 278(1-4), 532-537 (2005)
24. H.F. Liu, N. Xiang, S.J. Chua, Influence of $\mathrm{N}$ incorporation on In content in GaInNAs/GaNAs quantum wells grown by plasmaassisted molecular beam epitaxy. Appl. Phys. Lett. 89, 071905 (2006)

25. A. Albo, C. Cytermann, G. Bahir, D. Fekete, Utilizing the interface adsorption of nitrogen for the growth of high-quality GaInAsN/GaAs quantum wells by metal organic chemical vapor deposition for near infrared applications. Appl. Phys. Lett. 96, 141102 (2010)

26. Takuo Sasaki, Hidetoshi Suzuki, Masamitu Takahasi, Yoshio Ohshita, Itaru Kamiya, Masafumi Yamaguchi, X-ray reciprocal space mapping of dislocation-mediated strain relaxation during InGaAs/GaAs(001) epitaxial growth. J. Appl. Phys. 110, 113502 (2011)

27. J. Ishizaki, S. Goto, M. Kishida, T. Fukui, H. Hasegawa, Mechanism of multiatomic step formation during metalorganic chemical vapor deposition growth of GaAs on (001) vicinal surface studied by atomic force microscopy. Jpn. J. Appl. Phys. 33(1S), 721 (1994)

28. J. Li, T.F. Kuech, Evolution of surface structure during carbon doping in the metal-organic vapor-phase epitaxial growth of GaAs. J. Cryst. Growth 181(3), 171-180 (1997)

29. L. Auvray, H. Dumont, J. Dazord, Y. Monteil, J. Bouix, C. BruChevalier, AFM study and optical properties of GaAsN/GaAs epilayers grown by MOVPE. J. Cryst. Growth 221, 475-480 (2000)

30. Yeonjoon Park, Michael J. Cich, Rian Zhao, Petra Specht, Henning Feick, Eicke R. Weber, AFM study of lattice matched and strained InGaAsN layers on GaAs. Phys. B 308-310, 98-101 (2001)

31. A. Szyszka, M. Wośko, T. Szymański, R. Paszkiewicz, Surface topography analysis with application of roughness area dependence method. Ultramicroscopy 170, 77-85 (2016)

32. G.R. Mutta, S. Carapezzi, 2D scaling behavior of nanotextured GaN surfaces: a case study of hillocked and terraced surfaces. Appl. Surf. Sci. 447, 845-851 (2018)

33. E. Luna, A. Trampert, E.M. Pavelescu, M. Pessa, Nitrogenenhanced indium segregation in $(\mathrm{Ga}, \mathrm{In})(\mathrm{N}, \mathrm{As}) / \mathrm{GaAs}$ multiple quantum wells grown by molecular-beam epitaxy. New J. Phys. 9, 405 (2007)

34. D.J. Friedman, J.F. Geisz, S.R. Kurtz, J.M. Olson, R. Reedy, Nonlinear dependence of $\mathrm{N}$ incorporation on In content in GaInNAs. J. Cryst. Growth 195, 438-443 (1998)

35. Kudrawiec R(2004) Photoreflectance and photoluminescence investigations of nitrogen diluted III-V alloys and their low dimensional structures, $\mathrm{PhD}$ thesis, Wroclaw University of Science and Technology, Poland

36. J. Misiewicz, R. Kudrawiec, Contactless electroreflectance spectroscopy of optical transitions in low dimensional semiconductor structures. Opto-Electron. Rev. 20(2), 101-119 (2012)

37. J. Misiewicz, P. Sitarek, G. Sęk, R. Kudrawiec, Semiconductor heterostructures and device structures investigated by photoreflectance spectroscopy. Mater. Sci. 21(3), 263-320 (2003)

38. Prepared for publication

Publisher's Note Springer Nature remains neutral with regard to jurisdictional claims in published maps and institutional affiliations. 